\title{
Photocatalytic degradation of ampicillin using silver nanoparticles biosynthesised by Pleurotus ostreatus
}

\author{
Prabhjot S. Jassal *, Robinka Khajuria, Ronit Sharma, Pritam Debnath, \\ SONAL Verma, ABey Johnson, Sumit Kumar \\ Department of Biotechnology, Lovely Professional University, Phagwara, India
}

\begin{abstract}
The past few decades have witnessed a tremendous increase in the consumption of antibiotics worldwide. This rampant and unregulated use of antibiotics and their improper disposal has led to the accumulation of these drugs in the environment. This in turn has led to the emergence of antibiotic resistance in microbes and has become one of the most pressing global concerns in medicine, with highly resistant pathogens of many species proving difficult to treat. The aim of the study was to synthesise silver nanoparticles (Ag-NPs) using white rot fungus, Pleurotus ostreatus and assess its potential to carry out photocatalytic degradation of ampicillin. UV-Vis spectroscopy, Fourier transform infrared spectroscopy and transmission electron microscope have been used to characterize biosynthesized Ag-NPs. The photocatalytic degradation of ampicillin in aqueous solution by Ag-NPs was evaluated under natural sunlight. The effect of the operating conditions (contact time, Ag-NP concentration and initial ampicillin concentration) on the photocatalytic degradation was also investigated. The highest ampicillin degradation of $96.5 \%$ was observed after exposure of the solution (antibiotic + nanoparticles) for $4 \mathrm{~h}$ in sunlight. The maximum degradation was observed at an Ag-NP concentration of $5 \mathrm{ppm}$ at $\mathrm{pH}$. To the best of our knowledge, photocatalytic degradation of ampicillin using Ag-NPs synthesised by $P$. ostreatus has not been reported earlier.
\end{abstract}

Key words: ampicillin, FTIR, photocatalysis, silver nanoparticles (Ag-NPs), TEM

\section{Introduction}

In the past few years, global antibiotic consumption has increased to more than 70 billion standard units (SU), to which India alone contributes 13 billion SU (Gelband et al., 2015). The use of antibiotics is not just confined to human drugs alone; they are being extensively employed in animal breeding, analysis experiments, crop production, fish farming, and cultivation (Thurman et al., 2002). This excessive and unregulated usage of antibiotics has led to increased release of these drugs into the environment. Most antibiotics, once administered to animals or humans are not metabolized completely and eventually end up accumulating in the environment (Roose-Amsaleg and Laverman, 2016). Being recalcitrant in nature, they persist in the environment for a long time, thus creating conditions for micro-organisms to develop mechanisms to evade their antimicrobial actions. Antibiotic resistance occurring in bacterial pathogens has been recognized as a major public health concern affecting humans worldwide. The span of multidrug-resistant organisms is not only restricted to hospitals, but they are also often present in community settings (Munita et al., 2016). For instance, Indian water bodies have been reported to contain various antibiotics such as ampicillin, ciprofloxacin, erythromycin, and enrofloxacin (Balakrishna et al., 2016). Ampicillin belongs to the penicillin group of betalactam antibiotics and is capable of penetrating both Gram-positive and Gramnegative bacteria. Ampicillin acts as a competitive inhibitor of the enzyme transpeptidase, thereby affecting cell wall synthesis (Sharma et al., 2016).

The rampant and unregulated use of antibiotics and their improper disposal has become a significant challenge to the environment. Therefore, a two-pronged approach, based on a controlled use of antibiotics and their effective removal from contaminated sites, is needed. Although a number of methods have been reported for the degradation/removal of antibiotics, their efficiency

\footnotetext{
* Corresponding author: Department of Biotechnology, Lovely Professional University, Phagwara, India; e-mail: prabh_jyot2006@yahoo.co.in
} 
varies significantly. Readers may refer to the review article by Homem and Santos (2011), which provides a comparative analysis of different conventional methods like adsorption, coagulation, filtration, flocculation, sedimentation, and advanced oxidation processes used to remove antibiotics from the environment. However, the existing treatment methods for these contaminants focus only on the removal of the chemicals present and do not turn them into non-toxic wastes. The bottlenecks associated with these conventional methods of antibiotic degradation have led to an increased interest in the use nanoparticles (NPs) in the degradation of antibiotics (Guzmán et al., 2008). Photocatalytic detoxification is a process which amalgamates heterogeneous catalysis with solar technologies. Thus, the prime advantage of the photocatalytic process over other prevailing technologies is that it eliminates the need for further disposal. Furthermore, the application of NPs in phototocatalysis enhances photoredox chemistry, which in turn leads to increased photoactivity (Beydoun et al., 1999). The semiconductor material used in photocatalysis helps in the degradation of various organic pollutants present in water by the photoexcitation of electrons. Compared with traditional synthetic methods, biological systems employ novel techniques for the efficient production of nanomaterials. Fungi are valuable biological agents for synthesizing metal NPs. The use of fungi is potentially exciting as they secrete bulky amounts of enzymes and their biomass is easy to manage (Yehia and Al-Sheikh, 2014).

Pleurotus ostreatus is a temperate edible mushroom that can be grown on different agricultural wastes in a temperature range of $25-28^{\circ} \mathrm{C}$. P. ostreatus is known to produce various enzymes such as laccase, manganese peroxide, lignin peroxidases, xylanases etc. which catalyze various metabolic reactions such as substrate utilization and degradation of pollutants (Singh et al., 2017).

The aim of the study was to develop a suitable method with the potential to overcome all of the challenges associated with the improper disposal of antibiotics.

\section{Materials and methods}

\section{Maintenance of culture}

$P$. ostreatus was procured from ICAR, Directorate of Mushroom Research, Solan, India, and maintained on malt extract agar (at $25 \pm 2^{\circ} \mathrm{C}$ ). The composition of malt extract agar includes maltose $(12.75 \mathrm{~g} / \mathrm{l})$, dextrin
$(2.75 \mathrm{~g} / \mathrm{l})$, glycol $(2.35 \mathrm{~g} / \mathrm{l})$, peptone $(0.78 \mathrm{~g} / \mathrm{l})$, and agar $(15.0 \mathrm{~g} / \mathrm{l})$. For suspension culture, mycelium Plugs $(6 \mathrm{~mm})$ were excised using a sterile plug borer, and were inoculated in malt media. The culture was incubated at $25 \pm 2^{\circ} \mathrm{C}$ for $72 \mathrm{~h}$ at $100 \mathrm{rpm}$.

\section{Preparation of the cell filtrate}

The cell filtrate was prepared according to the modified method of Abdel-Hafez et al. (2016). Fungal biomass was harvested by centrifugation at $5000 \mathrm{rpm}$ for $5 \mathrm{~min}$ followed by washing with sterile distilled water to eliminate any traces of the media. The fungal biomass (about $2 \mathrm{~g})$ was then inoculated into sterile distilled water, incubated for $72 \mathrm{~h}$ at $25 \pm 2{ }^{\circ} \mathrm{C}$, and shaken at $100 \mathrm{rpm}$ for $72 \mathrm{~h}$. Whatman filter paper no. 1 was used for the filtration of the biomass and the obtained filtrate was stored at $4{ }^{\circ} \mathrm{C}$ till further use.

\section{Green synthesis of silver nanoparticles}

Silver nitrate was added to $100 \mathrm{ml}$ of cell filtrate to obtain a final concentration of $4 \mathrm{mM}$, followed by incubation for $72 \mathrm{~h}$ at $25 \pm 2{ }^{\circ} \mathrm{C}$ with shaking (100 rpm) under dark conditions. Silver nitrate solution without the cell filtrate was used as control and subjected to similar treatment. Nanoparticles were garnered by centrifugation at $5000 \mathrm{rpm}$ for $15 \mathrm{~min}$ and the pellets were freeze dried.

\section{UV-Visible spectroscopy}

The reduction of silver ions during silver nanoparticles (Ag-NP) synthesis was observed using UV-visible spectroscopy. The dried Ag-NPs were resuspended in sterile distilled water and subjected to a wavelength scan in the range of $300-700 \mathrm{~nm}$.

\section{Fourier-transform infrared spectroscopy (FTIR)}

Infrared measurements of Ag-NPs were carried out at room temperature on a Shimadzu 8400S FTIR spectrometer, armed with a potassium bromide (KBr) beam splitter. A thin layer of samples was dispersed in $\mathrm{KBr}$ in $100: 1$ ratio. This solid base was positioned in the sample holder and FTIR spectra were scanned in the range of $4000-500 \mathrm{~cm}^{-1}$.

\section{Transmission electron microscopy (TEM)}

The sample was sonicated for $15 \mathrm{~min}$ and the aqueous suspension of Ag-NPs was loaded on a carbon-coated copper grid. The solvent was permitted to evaporate 
under infrared light for 30 min (Devika et al., 2012). TEM measurements were performed at an accelerating voltage at $200 \mathrm{KV}$.

\section{Photocatalytic degradation of ampicillin}

\section{Effect of contact time}

Ampicillin (10 ppm) was mixed with freeze-dried Ag-NPs and incubated in sunlight (in March) for $6 \mathrm{~h}$ (between 10:00 am and 5:00 pm). Ampicillin solution without NPs served as control and was subjected to similar treatment. The samples were withdrawn after a an interval of $1 \mathrm{~h}$. Ampicillin assay was carried out every $\mathrm{h}$ to optimize the time for maximum degradation. It is worth specifying that all ampicillin assays in this paper were carried out according to the method described by Ahmed et al. (2004). Three ml of Folinciocalteu phenol reagent were added to $5 \mathrm{ml}$ of the sample, and incubated in a water bath at $95^{\circ} \mathrm{C}$ for $20 \mathrm{~min}$. The mixture was allowed to cool down and the absorbance was measured at $750 \mathrm{~nm}$. All experiments were carried out in triplicate. In addition, kindly note that in this paper the percentage of degradation has been calculated for every concentration of NPs using the method developed by Nosrati et al. (2012).

Percentage degradation $=((\mathrm{Co}-\mathrm{Ct}) / \mathrm{Co}) \times 100$

where, $\mathrm{Co}$ - initial absorbance, $\mathrm{Ct}$ - final absorbance after incubation.

\section{Effect of antibiotic concentration}

To assess the influence of the antibiotic concentration on the ability of nanoparticles to degrade the antibiotics, different concentrations of antibiotic $(10 \mathrm{ppm}$, $20 \mathrm{ppm}, 30 \mathrm{ppm}, 40 \mathrm{ppm}, 50 \mathrm{ppm}, 60 \mathrm{ppm}, 70 \mathrm{ppm}$, and $80 \mathrm{ppm}$ ) were incubated in sunlight for an optimized time with freeze-dried Ag-NPs. Antibiotic solutions without NPs served as controls and were subjected to similar treatment. Ampicillin assay was carried out and the percentage of degradation was calculated for every concentration. It is important to highlight that different concentrations of the antibiotic were evaluated to determine the optimized concentration at which maximum degradation occurred.

\section{Effect of NP concentration}

The optimized ampicillin concentration was treated with different concentrations of freeze-dried Ag-NPs (1 ppm, 3 ppm, 7 ppm, 9 ppm, 11 ppm, 13 ppm, 15 ppm, and $17 \mathrm{ppm}$ ) and subjected to photocatalytic degradation for an optimized contact time. The antibiotic solution without NPs served as control and was subjected to similar treatment. Ampicillin assay was carried out and the percentage of degradation was calculated for every concentration of NPs. It is important to highlight that different concentrations of Ag-NPs were used to determine the optimized concentration at which maximum degradation of antibiotics occurred.

\section{Effect of $p H$}

Ampicillin solutions at the optimized concentration were prepared for different $\mathrm{pH}$ values ranging from 4 to 9 . The samples were treated with the optimized NP concentration and subjected to photocatalytic degradation for the optimized contact time. The antibiotic solution without NPs served as control and was subjected to similar treatment. Ampicillin assay was carried out and the percentage of degradation was calculated at every $\mathrm{pH}$ value.

\section{Statistical analysis}

A statistical analysis of the obtained results was performed to evaluate the significance of the variables over the range of antibiotic degradation. One-way and two-way ANOVA were used with the statistical software package Graphpad PRISM 5.0.

\section{Results and discussion}

The purpose of the present study was to assess the influence of different parameters, such as the reaction time, the concentration of antibiotics and NPs, and the $\mathrm{pH}$ of the solution on the degradation of ampicillin. An in-depth analysis of the degradation mechanism revealed that photocatalytic activity plays a prominent role in the reduction of toxic wastes, as holes $\left(\mathrm{H}^{+}\right)$in aqueous media get trapped by water molecules and hydroxyl radicals $\left(\mathrm{OH}^{-}\right)$are generated. These radicals act as strong oxidizing agents and oxidize organic pollutants to water and gaseous products (Anjum et al., 2016).

\section{Green synthesis of NPs}

The preliminary indication of NP synthesis was the alteration in fungal suspension color. In the present study, a change in color to dark brown was observed within $72 \mathrm{~h}$ of incubation of silver nitrate with cell filtrate of $P$. ostreatus (Fig. 1). However, no color change 


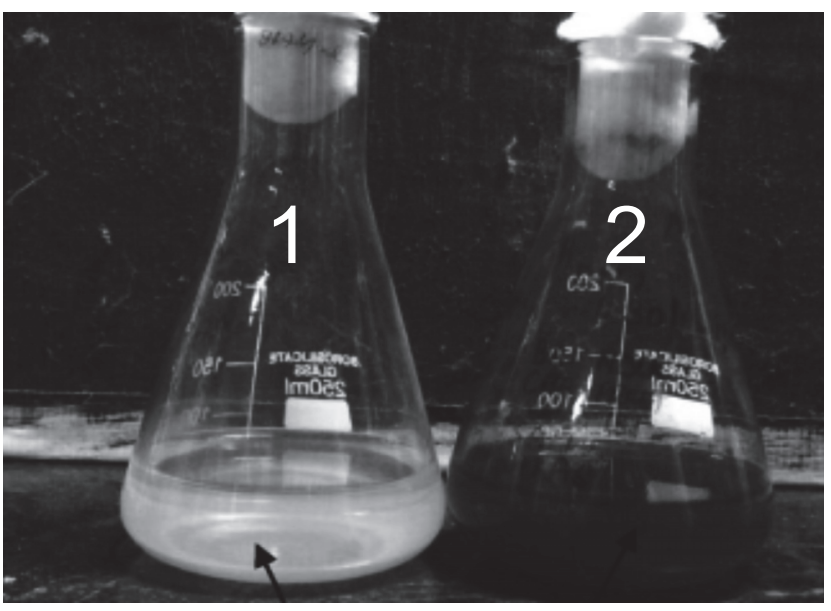

Fig. 1. The synthesis of AgNPs by Pleurotus ostreatus resulting in a change of the color of the suspension 1) before and 2) after synthesis

was observed in the case of negative control (silver nitrate solution alone). A similar change in color, from a colorless solution to dark brown, indicating the synthesis of Ag-NPs by the fungus Verticillium was reported by Mukherjee et al. (2001). Similarly, the synthesis of Ag-NPs by Aspergillus fumigatus, Candida albicans, Penicillium italicum, Syncephalastrum racemosum, Fusarium oxysporum, and Aspergillus ochraceus resulted in a dark brown colour of the suspensions (Magdi et al., 2014). Kathiresan et al. (2009) also reported a change in color to dark brown as a result of the synthesis of Ag-NPs by Penicillium fellutanum isolated from mangrove root-soil. This change in color is attributed to the surface plasmon resonance of the deposited Ag-NPs. Ag-NPs absorb radiation in the visible region of the electromagnetic spectrum; the excitation of surface plasmons (vibrations) is responsible for the prominent yellow-brown color of Ag-NPs in various media (Vigneshwaran et al. 2007).

\section{UV-Visible spectroscopy}

To validate the formation and evaluate the stability of Ag-NPs, UV-Visible spectroscopy of the colloidal solution was carried out. A typical surface plasmon absorption band was observed around $435 \mathrm{~nm}$ in the tested sample with a corresponding band gap of $2.85 \mathrm{eV}$; no absorption band was observed in the control (Fig. 2). The formation of Ag-NPs from the fungus biomass can be attributed to the trapping of silver ions at the surface of fungal cells followed by their reduction by the enzymes present in the fungal system. Devaraj et al. (2013) also reported

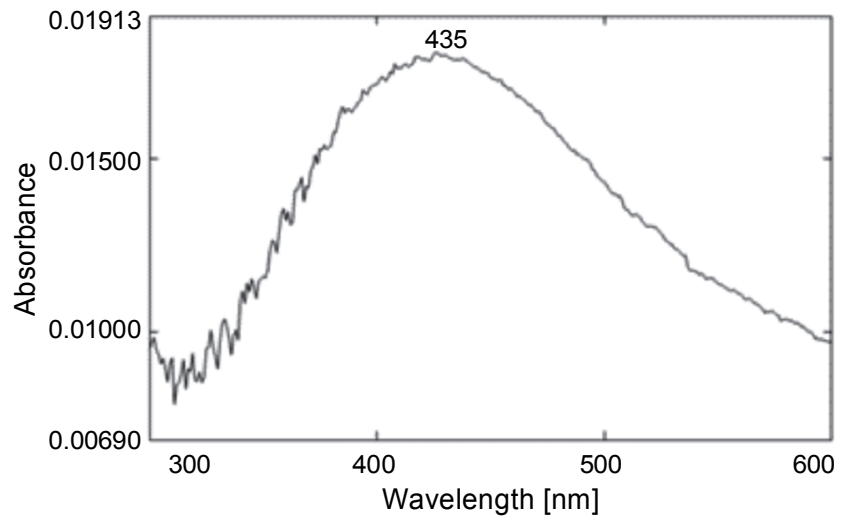

Fig. 2. UV-visible absorption spectra of Ag-NPs

a silver surface plasmon resonance band of $430 \mathrm{~nm}$ for NPs synthesised using cannonball leaves. A $414 \mathrm{~nm}$ resonance peak of the surface plasmon has been reported for Ag-NPs synthesised by Urtica dioica linn leaves. Previous studies suggest that an SPR peak located between $410 \mathrm{~nm}$ and $450 \mathrm{~nm}$ has been observed for Ag-NPs and might be attributed to spherical NPs (Jyoti et al., 2016). Ahmad et al. (2004) reported a sharp SPR peak at $413 \mathrm{~nm}$ for Ag-NPs synthesised by Fusarium oxysporum. Ag-NPs from Schizophyllum radiatum exhibited an SPR peak at $435 \mathrm{~nm}$ (Metuku et al., 2013). Similarly, Yehia and Al-Sheikh (2014) reported a maximum absorbance at $435 \mathrm{~nm}$ for Ag-NPs synthesised by $P$. ostreatus.

\section{FTIR spectroscopy}

FTIR analysis was done to characterize the functional groups associated with NPs. As shown in Figure 3, the spectrum band at $1645 \mathrm{~cm}^{-1}$ can be attributed to $-\mathrm{C}=\mathrm{O}$ stretch vibrations and these stretch vibrations are present in the amide linkages of the proteins. The peaks at $3441.2 \mathrm{~cm}^{-1}$ and $2926.1 \mathrm{~cm}^{-1}$ pertain to the stretching vibrations of primary and secondary amines, respectively (Vigneshwaran et al., 2007). The two bands observed near $1342 \mathrm{~cm}^{-1}$ and $1037 \mathrm{~cm}^{-1}$ can be assigned to the $\mathrm{C}-\mathrm{N}$ stretching vibrations of aromatic and aliphatic amines, respectively. The molecular vibrational positions of these bands are in agreement with earlier studies on native proteins by Labrenz et al. (2000). FTIR measurements indicate (data not shown) that the secondary structures of proteins are not affected by their interaction with silver ions or NPs. However, there are no data to confirm/prove this.

The carbonyl groups present in amino acids, peptides and proteins residues can strongly bind to metals. There- 


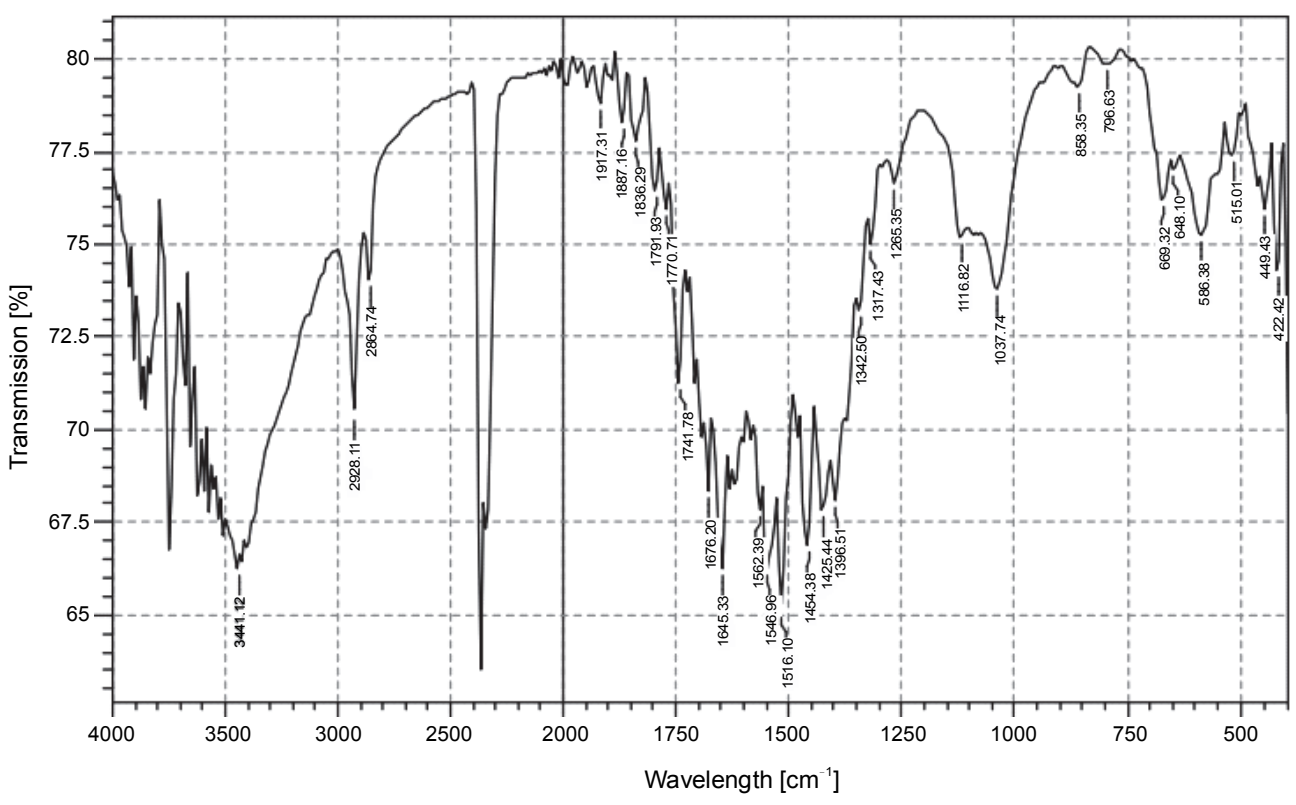

Fig. 3. FTIR spectra of Ag-NPs
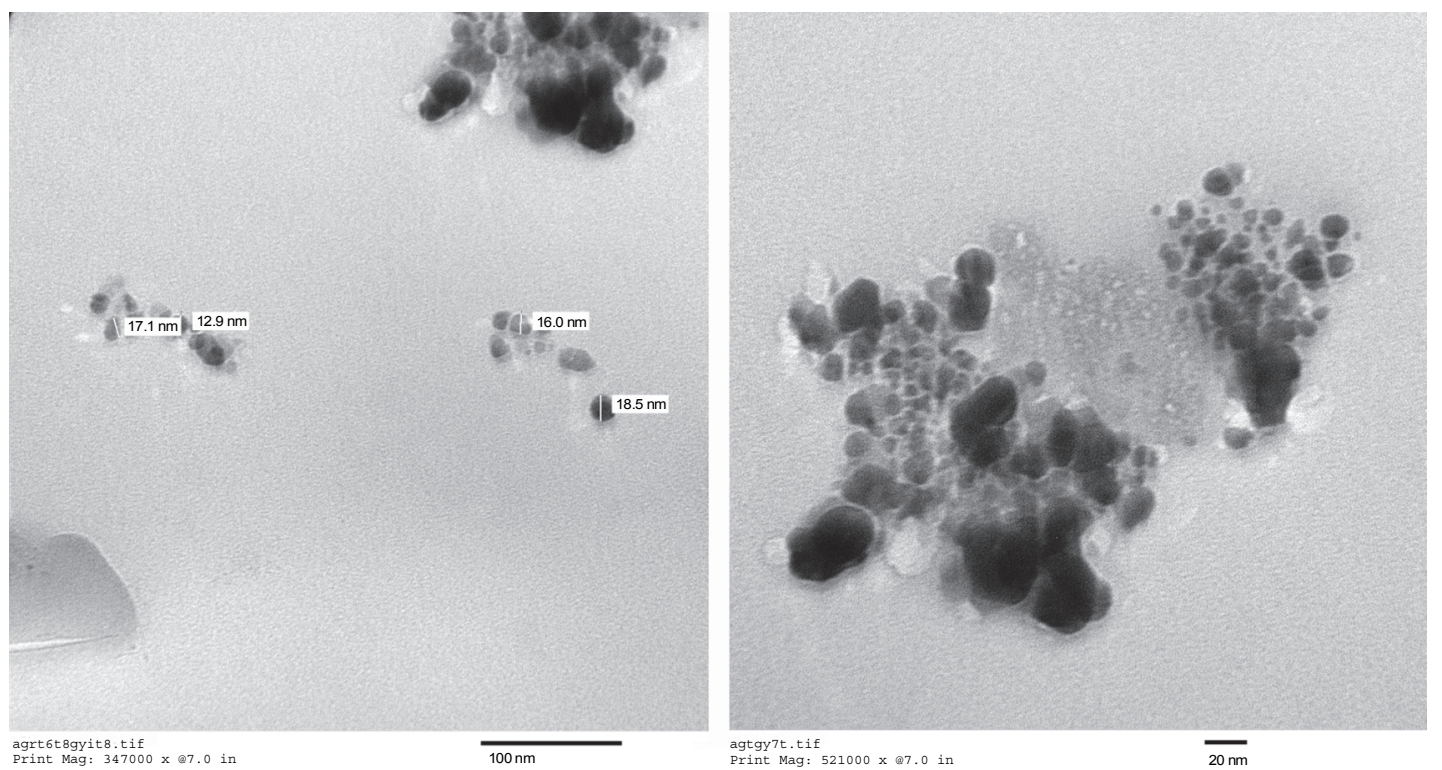

Fig. 4. TEM images of biogenic silver nanoparticles

fore, proteins may act as capping agents of Ag-NPs, preventing agglomeration and stabilizing the particles within the medium (Abdel-Hafez et al., 2016). Proteins can bind to NPs either via free amine groups or cysteine residues or by the electrostatic interactions of negatively charged carboxylate groups of enzymes present in the cell wall of mycelia and therefore, stabilize the NPs (Magdi et al., 2014).

\section{Transmission electron microscopy (TEM)}

The TEM analysis allowed further understanding of the size and shape of Ag-NPs. TEM images recorded from the Ag-NPs, placed on carbon-coated Ag-NPs, revealed their polydispersed and spherical nature (Fig. 4). Most of the NPs shown in the micrograph are in the range of $12.9 \mathrm{~nm}$ to $18.5 \mathrm{~nm}$. 


\section{Photocatalytic degradation of ampicillin}

\section{Effect of contact time}

To assess the optimal time for the antibiotic (ampicillin) degradation, Ag-NPs were incubated with ampicillin $(10 \mathrm{ppm})$ in sunlight for $6 \mathrm{~h}$ with an average light intensity of 940-1000 Lux. The samples were taken regularly at an interval of $1 \mathrm{~h}$. The antibiotic solution without NPs served as control and was subjected to similar treatment. Although antibiotic degradation was observed in both controls and samples, the presence of Ag-NPs accelerated the degradation process, with a maximum degradation of $96.5 \%$ observed after $4 \mathrm{~h}$ of incubation as compared to $69.3 \%$ in the control. A substantial degradation of $75.5 \%$ was observed after just 1 hour of incubation as compared to $35 \%$ degradation in the control. Although there was a gradual increase in the antibiotic degradation with increase in contact time, no significant increase was observed after $4 \mathrm{~h}$ of incubation. This can be attributed to the decreased intensity of sunlight with time (Fig. 5). Exposure to UV rays in sunlight leads to the excitation of electrons in Ag-NPs from the valence band to a conduction band. The holes generated in the valence band of the semiconductor, along with hydroxyl radicals, may lead to direct or indirect oxidation of ampicillin. Indirect oxidation may be the result of the generation of hydroxide reactive radicals $(\mathrm{OH})$ via the reaction of holes in the valence band with water or hydroxide anions $\left(\mathrm{OH}^{-}\right)$. Primary photo-products formed due to the electron-hole transfer undergo further transformational steps to form a final photo-product, thereby leading to complete antibiotic degradation. The rate of degradation therefore, decreases with the decrease in light intensity, which leads to the decrease in the energy of the photon, moreover the overall energy introduced into the photocatalytic process is reliant on light intensity (Jodat and Jodat, 2013). It is worth mentioning here that the extent of degradation achieved during $4 \mathrm{~h}$ of incubation in natural sunlight is of great significance for developing an efficient and quick method for antibiotic remediation. In a work reported by Nosrati et al., (2012), 41\% ampicillin degradation was achieved after $120 \mathrm{~min}$ of incubation in sunlight with $\mathrm{ZnO} /$ polyamine nanocomposites. In another report by Elmolla and Chaudhuri (2010), 96\% ampicillin degradation was observed upon incubation with $\mathrm{ZnO} N \mathrm{NP}$ for $300 \mathrm{~min}$ under UV lamp (365 nm). The biggest advantage of using NPs

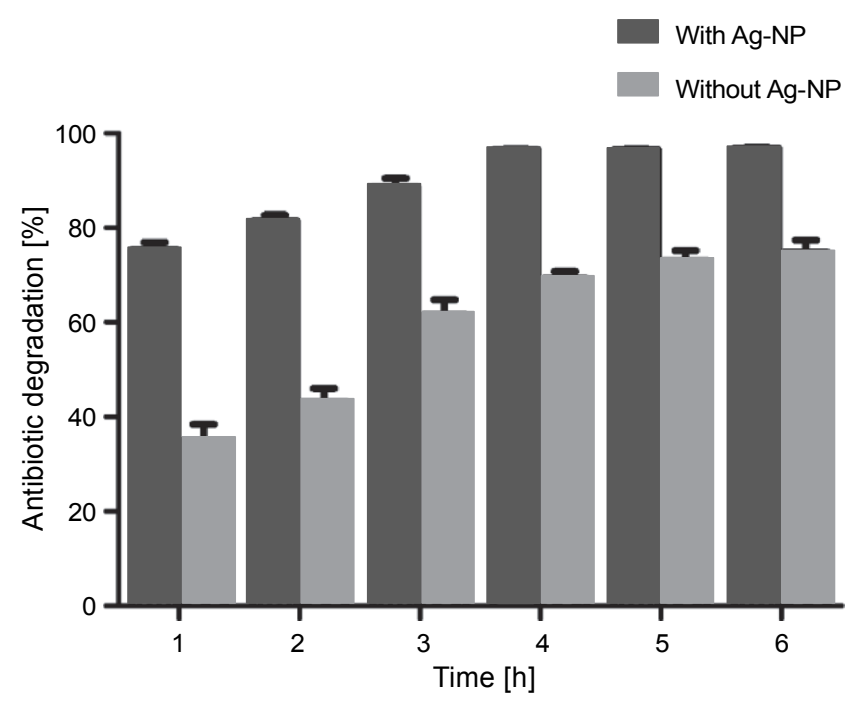

Fig. 5. Effect of contact time on ampicillin degradation

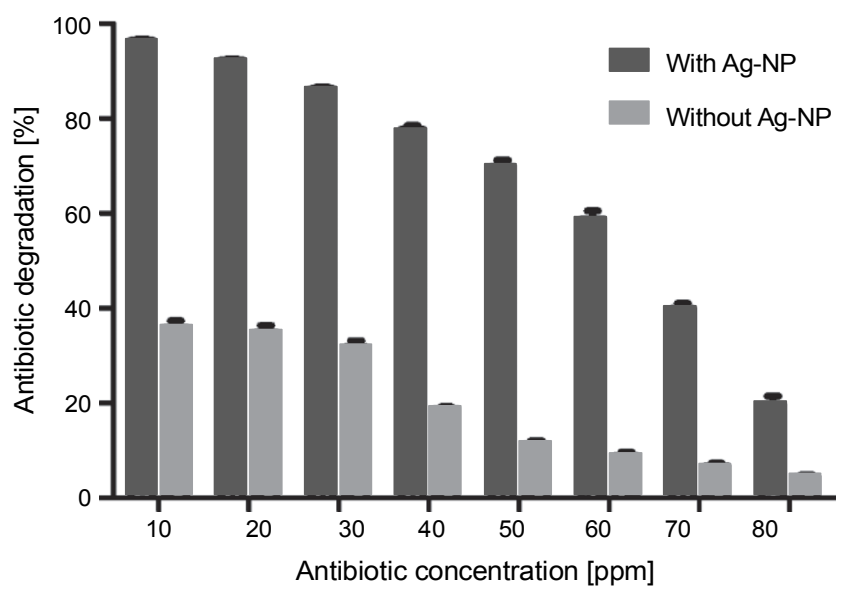

Fig. 6. Effect of initial ampicillin concentration on degradation

as photocatalysts is the reduction in treatment time compared to that with conventional biological methods which have been reported to take around 7-14 days (Singh et al., 2017; Kumar et al., 2013; Prieto et al., 2011).

Two way ANOVA revealed that both contact time and NPs have a significant effect on antibiotic degradation. This effect was found to be in synergy as revealed by the interaction $\mathrm{p}$ value $(P<0.0001)$.

\section{Effect of initial antibiotic concentration}

To evaluate the extent of photocatalytic degradation at higher concentrations of ampicillin, antibiotics of different concentrations (10-80 ppm) with Ag-NPs were exposed to sunlight for $4 \mathrm{~h}$. The antibiotic solution without NPs served as control and was subjected to similar treatment. As observed previously, ampicillin degrada- 
Table 1. Two way ANOVA for effect of time and presence of NPs on antibiotic degradation

\begin{tabular}{l|c|c|c|c|c|c}
\hline \multicolumn{1}{c|}{ Source of variation } & Df & Sum of squares & Mean square & $F$ & $P$-value & Significant? \\
\hline Interaction & 5 & 445.2 & 89.04 & 21.38 & $<0.0001$ & yes \\
\hline Presence o NPs & 1 & 7989 & 7989 & 1918 & $<0.0001$ & yes \\
\hline Time & 5 & 4961 & 992.3 & 238.2 & $<0.0001$ & yes \\
\hline Residual & 24 & 99.97 & 4.166 & & & \\
\hline
\end{tabular}

Table 2. Two way ANOVA for antibiotic concentration and presence of NPs on antibiotic degradation

\begin{tabular}{l|c|c|c|c|c|c}
\hline \multicolumn{1}{c|}{ Source of variation } & Df & Sum of squares & Mean square & $F$ & $P$-value & Significant? \\
\hline Interaction & 7 & 2733 & 390.4 & 337.9 & $<0.0001$ & yes \\
\hline Presence of NPs & 1 & 28290 & 28290 & 24490 & $<0.0001$ & yes \\
\hline Antibiotic concentration & 7 & 16130 & 2304 & 1994 & $<0.0001$ & yes \\
\hline Residual & 32 & 36.97 & 1.155 & & & \\
\hline
\end{tabular}

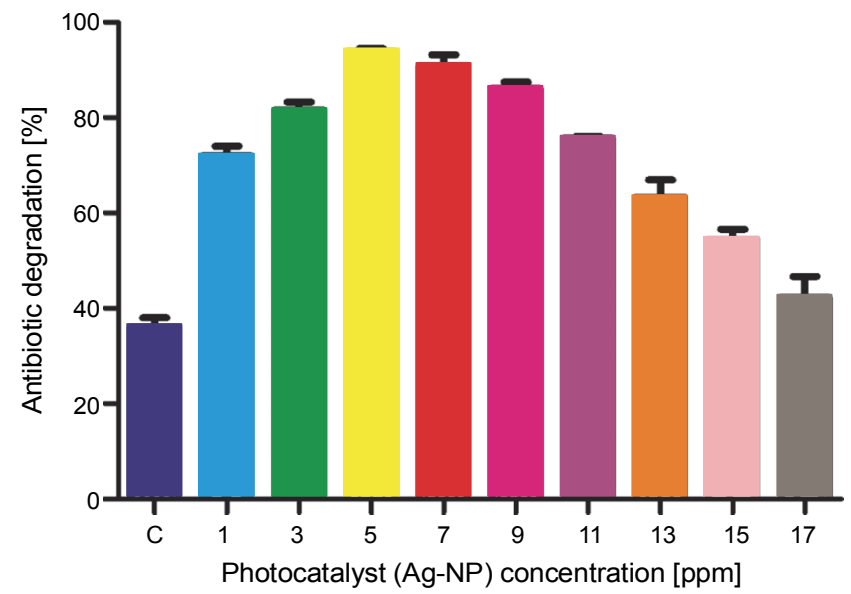

Fig. 7. Effect of photocatalyst concentration on antibiotic degradation

tion was observed in both cases, though the presence of Ag-NPs enhanced the process. The maximum degradation of $96.5 \%$ was observed at $10 \mathrm{ppm}$ of ampicillin; however, a considerable amount of degradation, $69.9 \%$ and $58.8 \%$, was observed at $50 \mathrm{ppm}$ and $60 \mathrm{ppm}$, respectively, in contrast to $11.2 \%$ and $8.9 \%$ degradation in controls (Fig. 6). A similar degradation pattern has been reported for the degradation of ampicillin by $\mathrm{ZnO} /$ polyamine nanocomposites by Nosrati et al. (2012). The decrease in the degradation level at high concentrations can be attributed to the fact that the adsorbed reactant molecules lodge in all the active sites of the catalyst. Moreover, with an increase in the target pollutant (antibiotic) concentration, more antibiotic molecules become adsorbed on the surface of the catalyst. Therefore, the

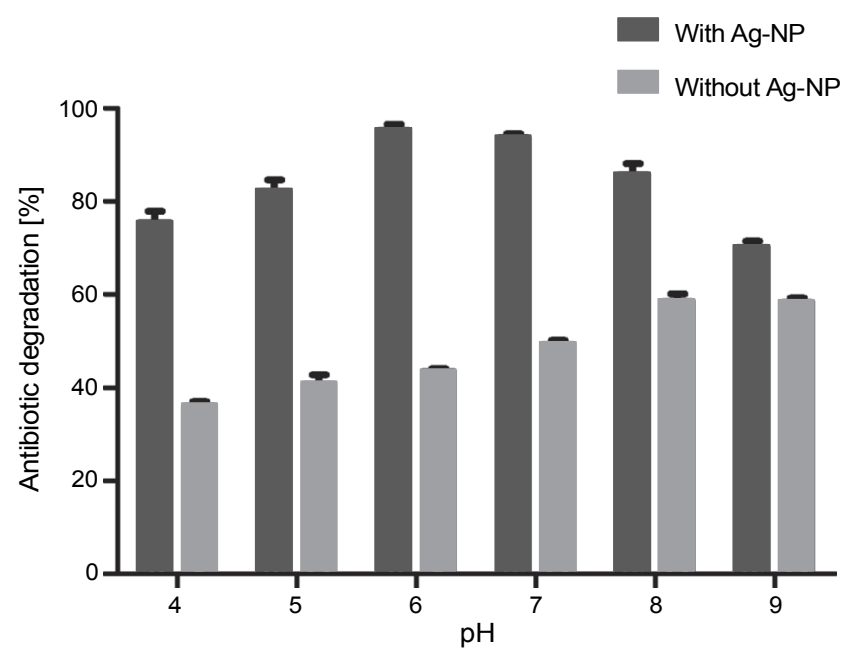

Fig. 8. Effect of $\mathrm{pH}$ on the photocatalytic degradation of antibiotics

requirement of the reactive species $(\mathrm{OH})$ for the degradation of the pollutants also increases. As the concentration of the target pollutant increases, the pollutant molecules absorb more light, and therefore, photons never reach the catalyst surface, and so the photodegradation rate decreases (Jodat and Jodat, 2013).

Statistical analysis revealed that the initial antibiotic concentration had a profound effect on the antibiotic degradation rate $(P<0.0001)$. Also, the presence of NPs affected antibiotic degradation in a significant way $(P<0.0001)$. Antibiotic degradation was affected by the presence of NPs over the entire range of antibiotic concentration as indicated by a small interaction $P$-value (Table 2). 
Table 3. One-way ANOVA for nanoparticle concentration on antibiotic degradation

\begin{tabular}{l|c|c|c}
\hline \multicolumn{1}{c|}{ One-way analysis of variance } & & \\
\hline$P$-value & $<0.0001$ & & \\
\hline Are means significantly different? $(P<0.05)$ & yes & & \\
\hline ANOVA Table & SS & df & MS \\
\hline Varying NP concentration (between columns) & 7160 & 8 & 895.0 \\
\hline Residual (within columns) & 103.3 & 18 & 5.739 \\
\hline Total & 7263 & 26 & \\
\hline
\end{tabular}

Table 4. Two-way ANOVA for $\mathrm{pH}$ and the presence of NP on antibiotic degradation

\begin{tabular}{l|c|c|c|c|c|c}
\hline \multicolumn{1}{c|}{ Source of variation } & Df & Sum of squares & Mean square & $F$ & $P$-value & Significant? \\
\hline Interaction & 5 & 1520 & 303.9 & 109.3 & $<0.0001$ & yes \\
\hline Presence of nanoparticles (NP) & 1 & 11650 & 11650 & 4189 & $<0.0001$ & yes \\
\hline $\mathrm{pH}$ & 5 & 1236 & 247.2 & 88.90 & $<0.0001$ & yes \\
\hline Residual & 24 & 66.73 & 2.780 & & & \\
\hline
\end{tabular}

\section{Effect of photocatalyst concentration}

To observe the effect of Ag-NP concentration on ampicillin degradation efficiency, a range of concentrations (1-19 ppm) was tested. Antibiotic degradation increased with increase in NP concentration, with maximum degradation (93.8\%) achieved at 5 ppm compared to $36 \%$ in the control sample without Ag-NPs (Fig. 7). Interestingly, a further increase in photocatalyst concentration was associated with a decrease in the degradation level of ampicillin. Initially, with increase in photocatalyst concentration, an increase in antibiotic degradation was observed, and it can be attributed to the increase in the available catalyst surface. However, increasing the amount of catalyst beyond a threshold concentration led to decreased antibiotic removal due to the increased turbidity of the solution which caused scattering of light, thus inhibiting its path through the solution. Similar antibiotic degradation efficiencies have been reported by various researchers (Nosrati et al., 2012; Jodat and Jodat, 2013; Elmolla and Chaudhuri, 2010).

One-way ANOVA revealed a strong correlation between photocatalyst concentration and antibiotic degradation $(P<0.0001)$ as depicted in Table 3 .

\section{Effect of $p H$}

The incubation of ampicillin with Ag-NPs in sunlight for $3 \mathrm{~h}$ at different $\mathrm{pH}$ values revealed a gradual increase in the percentage of degradation with increase in $\mathrm{pH}$ value. The maximum degradation (95.2\%) was achieved at $\mathrm{pH} 6$, while at higher $\mathrm{pH}$ values a decrease in antibiotic degradation was observed (Fig. 8). Generally, the effect of $\mathrm{pH}$ on antibiotic degradation depends on the properties of both the antibiotic and the NPs. When $\mathrm{pH}$ increased, the overall surface charge of Ag-NPs changed from highly negative to less negative. A positive surface charge is typically not seen on Ag-NPs at any $\mathrm{pH}$ unless engineered with a surface coating for this purpose (Badawy et al., 2010). On the other hand, ampicillin exists in cationic form at acidic $\mathrm{pH}$ and in an anionic form at alkaline $\mathrm{pH}$. Since at acidic $\mathrm{pH}$, ampicillin has a positive charge and the photocatalyst has a negative charge, it favours the absorption of ampicillin molecules on the photocatalyst surface, thereby exhibiting high degradation efficiency. At alkaline $\mathrm{pH}$, both ampicillin and the Ag-NPs are negatively charged, which leads to the generation of repulsive forces between the antibiotic and the catalyst. However, a substantial degradation is still observed at alkaline $\mathrm{pH}$, and it can be attributed to the hydrolysis of the antibiotic due to the instability of the $\beta$-lactam ring at $\mathrm{pH}>7.3$ (Rozas et al., 2010).

Two-way ANOVA suggested a significant effect of $\mathrm{pH}$ and the presence of NPs, individually, on the degradation of the antibiotic $(P<0.0001)$. Also, the $P$-value of interactions $(P<0.0001)$ revealed that NPs were effective in the degradation of the antibiotic in the entire range of $\mathrm{pH}$ under study (Table 4 ). 


\section{Conclusions}

Cell filtrate of $P$. ostreatus mediated Ag-NPs were synthesized in the range $12.9-18.5 \mathrm{~nm}$. The synthesized NPs exhibited a maximum ampicillin degradation of $96.5 \%$ after a $4 \mathrm{~h}$ incubation in sunlight. Likewise, other optimized parameters for maximum degradation included Ag-NP concentration of $5 \mathrm{ppm}$, ampicillin concentration of $10 \mathrm{ppm}$ and $\mathrm{pH} 6$. Additionally, to the best of our knowledge, photocatalytic degradation of ampicillin using Ag-NPs produced by $P$. ostreatus has not been reported before.

\section{Acknowledgments}

The authors are grateful to the Chancellor, Lovely Professional University for providing the necessary facilities and funds for undertaking this research.

\section{References}

Abdel-Hafez S.I.I., Nafady N.A., Abdel-Rahim I.R, Abeer M., José-Antonio S., Mohamed D.M.A. (2016) Assessment of protein silver nanoparticles toxicity against pathogenic Alternaria solani. 3 Biotech. 6: 199. https://doi.org/10.1007/ s13205-016-0515-6.

Ahmad A.S., Rahman N., Islam F. (2004) Spectrophotometric determination of ampicillin, amoxycillin, and carbenicillin using folin-ciocalteu phenol reagent. J. Analyt. Chem. 59(2): 119-123. https://doi.org/10.1023/B:JANC. 0000014736. 59554.5c.

Anjum M., Miandad R., Waqas M., Gehany F., Barakat M.A. (2016) Remediation of wastewater using various nanomaterials. Arabian J. Chem. https://doi.org/10.1016/ j.arabjc.2016.10.004.

Badawy A.M.E.L., Luxton T.P., Silva R.G., Scheckel K.G., Suidan M.T. (2010) Impact of environmental conditions ( $\mathrm{pH}$, ionic strength, and electrolyte type) on the surface charge and aggregation of silver nanoparticles suspensions. Environ. Sci. Technol. 44(4): 1260-1266. https:// doi.org/10.1021/es902240k.

Balakrishna K., Rath A., Praveenkumarreddy Y., Guruge K.S., Subedi B. (2016) A review of the occurrence of pharmaceuticals and personal care products in Indian water bodies. Ecotoxicol. Environ. Safety 137: 113-120. https:// doi.org/10.1016/j.ecoenv.2016.11.014.

Beydoun D., Amal R., Low G., McEvoy S. (1999) Role of nanoparticles in photocatalysis. J. Nanopart. Res. 1(4): 439-458. https://doi.org/10.1023/A:1010044830871.

Devaraj P., Kumari P., Aarti C., Renganathan A. (2013) Synthesis and characterization of silver nanoparticles using cannonball leaves and their cytotoxic activity against $M C F$ 7 cell line. J. Nanotechnol. https://doi.org/10.1155/2013/ 598328.

Devika R., Elumalai S., Manikandan E., Eswaramoorthy D. (2012) Biosynthesis of silver nanoparticles using Pleurotus ostreatus and their antibacterial activity. Sci. Rep. Bergqvist 1(11): 1-5. https://doi.org/10.4172/scientific reports.5.

Elmolla E.S., Chaudhuri M. (2010) Photocatalytic degradation of amoxicillin, ampicillin and cloxacillin antibiotics in aqueous solution using UV/TiO2 and UV/H2O2/TiO2 photocatalysis. Desalination 252(1-3): 46-52. https://doi.org/ 10.1016/j.desal.2009.11.003

Gelband H., Miller-Petrie M., Suraj P., Gandra S., Levinson J., Barter D., White A.R.L. (2015) The state of the world's antibiotics 2015. Centre for Disease Dynamics, Economics \& Policy, CDDEP: Washington, D.C: $1-84$. https://doi.org/ 10.1016/S1473-3099(13)70318-9.

Guzmán M.G.M., Dille J., Godet S. (2008) Synthesis of silver nanoparticles by chemical reduction method and their antibacterial activity. Intern. Sholarly Sci. Res. Innovat. 2(7): 91-98. https://doi.org/10.1007/s11814-010-0067-0.

Homem V., Santos L. (2011) Degradation and removal methods of antibiotics from aqueous matrices - a review. J. Environ. Manag. 92(10): 2304-2347. https://doi.org/ 10.1016/j.jenvman.2011.05.023.

Jodat A., Jodat A. (2013) Photocatalytic degradation of chloramphenicol and tartrazine using $\mathrm{Ag} / \mathrm{TiO}_{2}$ nanoparticles. Desalinat. Water Treat. 52(13-15). https://doi.org/ 10.1080/19443994.2013.794115.

Jyoti K., Baunthiyal M., Singh A. (2016) Characterization of silver nanoparticles synthesized using Urtica dioica Linn. leaves and their synergistic effects with antibiotics. J. Radiat. Res. Appl. Sci. 9(3): 217-227. https://doi.org/ 10.1016/j.jrras.2015.10.002.

Kathiresan K., Manivannan S., Nabeel M.A., Dhivya B. (2009) Studies on silver nanoparticles synthesized by a marine fungus, Penicillium fellutanum isolated from coastal mangrove sediment. Coll. Surf. B: Biointerfaces 71(1): 133-137. https://doi.org/10.1016/j.colsurfb.2009.01.016.

Kumar R.R., Park B.J., Jeong H.R., Lee J.T., Cho J.Y. (2013) Biodegradation of B-lactam antibiotic ampicillin by white rot fungi from aqueous solutions. J. Pure Appl. Microbiol. 7(4): 3163-3169.

Labrenz M., Druschel G.K., Thomsen-Ebert T., Gilbert B., Welch S.A. et al. (2000) Formation of sphalerite $(Z n S)$ deposits in natural biofilms of sulfate-reducing bacteria. Science 290: 1744-1747. https://doi.org/10.1126/science. 290.5497.1744.

Magdi H.M., Mourad M.H.E., Abd El-Aziz M.M. (2014) Biosynthesis of silver nanoparticles using fungi and biological evaluation of mycosynthesized silver nanoparticles. Egyptian J. Exp. Biol. 10(1): 1-12.

Metuku R.P., Pabba S., Burra S., Hima Bindu N.S.V.S.S.S.L., Gudikandula K., Singara Charya M.A. (2013) Biosynthesis of silver nanoparticles from Schizophyllum radiatum $\mathrm{HE}$ 863742.1: their characterization and antimicrobial activity. 3 Biotech. 227-234. https://doi.org/10.1007/s13205-0130138-0.

Mukherjee P., Ahmad A., Mandal D., Senapati S., Sainkar S.R., Khan M.I., Sastry M. (2001) Fungus-mediated synthesis of silver nanoparticles and their immobilization in 
the mycelial matrix: a novel biological approach to nanoparticle synthesis. Nano Lett. 1(10): 515-519. https:// doi.org/10.1021/n10155274.

Munita M.J., Arias C.A. (2016) Mechanisms of antibiotic resistance. Microbiol. Spectrum 4(2): 1-24. https://doi.org/ 10.1128/microbiolspec.VMBF-0016-2015.

Nosrati R., Olad A., Maramifar R. (2012) Degradation of ampicillin antibiotic in aqueous solution by $\mathrm{ZnO} /$ polyaniline nanocomposite as photocatalyst under sunlight irradiation. Environ. Sci. Pollut. Res. 19(6): 2291-2299. https:// doi.org/10.1007/s11356-011-0736-5.

Prieto A., Möder M., Rodil R., Adrian L., Marco-urrea E. (2011) Degradation of the antibiotics norfloxacin and ciprofloxacin by a white-rot fungus and identification of degradation products. Bioresource Technol. 102(23): 10987-10995. https:// doi.org/10.1016/j.biortech.2011. 08.055.

Roose-Amsaleg C., Laverman A.M. (2016) Do antibiotics have environmental side-effects? Impact of synthetic antibiotics on biogeochemical processes. Environ. Sci. Pollution Res. 23(5): 4000-4012. https://doi.org/10.1007/s11356-0154943-3.

Rozas O., Contreras D., Mondaca M.A., Mansilla H.D. (2010) Experimental design of Fenton and photo-Fenton reactions for the treatment of ampicillin solutions. J. Hazardous Mat. 177(1-3): 1025-1030. https://doi.org/10.1016/j.jhazmat. 2010.01.023.

Singh S.K., Khajuria R., Kaur L. (2017) Biodegradation of ciprofloxacin by white rot fungus Pleurotus ostreatus. 3Biotech 7: 69. https://doi.org/10.1007/s13205-017-0684-y.

Sharma S.K., Singh L., Singh S. (2013) Comparative study between Penicillin and Ampicillin. Schol. J. Appl. Med. Sci. 1(4): 291-294.

Thurman E.M., Dietze J.E., Scribner E.A. (2002) Occurrence of antibiotics in water from fish hatcheries. U.S. Geol. Survey Fact Sheet 120-02, (November).

Vigneshwaran N., Ashtaputre N.M., Varadarajan P.V., Nachane R.P., Paralikar K.M., Balasubramanya R.H. (2007) Biological synthesis of silver nanoparticles using the fungus Aspergillus flavus. Mater. Lett. 61(6): 1413-1418. https:// doi.org/10.1016/j.matlet.2006.07.042.

Yehia R.S., Al-Sheikh H. (2014) Biosynthesis and characterization of silver nanoparticles produced by pleurotus ostreatus and their anticandidal and anticancer activities. World J. Microbiol. Biotechnol. 30(11): 2797-2803. https:// doi.org/10.1007/s11274-014-1703-3. 